\title{
PERSPECTIVE
}

\section{A Model for Advancing Scale-Up of Complex Interventions for Vulnerable Populations: the ALACRITY Center for Health and Longevity in Mental IIIness}

\author{
Emma E. McGinty, PhD, $M S^{\top}$, Karly A. Murphy, MD, MHS ${ }^{2}$, Arlene T. Dalcin, $M S, R D^{2}$, \\ Elizabeth A. Stuart, $P h D^{3}$, Nae-Yuh Wang, $P h D^{2}$, Faith Dickerson, $P h D, M_{P H}{ }^{4}$, \\ Kim Gudzune, MD, MPH², Gerald Jerome, $P h D^{5}$, David Thompson, DNSc, $M S^{6}$, \\ Bernadette A. Cullen, MB, BCh, MRCPsych', Joseph Gennusa, PhD, RD, LDN², \\ Amy M. Kilbourne, $P h D, M P H^{8}$, and Gail L. Daumit, $M D, M H S^{2}$
}

\begin{abstract}
'Department of Health Policy and Management, Johns Hopkins Bloomberg School of Public Health, Baltimore, MD, USA; ${ }^{2}$ Division of General Internal Medicine, Johns Hopkins School of Medicine, Baltimore, MD, USA; ${ }^{3}$ Department of Mental Health, Johns Hopkins Bloomberg School of Public Health, Baltimore, MD, USA; ${ }^{4}$ Sheppard Pratt Health System, Baltimore, MD, USA; ${ }^{5}$ Department of Kinesiology, Towson University, Towson, MD, USA; ${ }^{6}$ Department of Anesthesiology and Critical Care and Armstrong Institute for Patient Safety and Quality, Johns Hopkins School of Medicine, Baltimore, MD, USA; ${ }^{7}$ Department of Psychiatry \& Behavioral Sciences, Johns Hopkins School of Medicine, Baltimore, MD, USA; ${ }^{8} \mathrm{Health}$ Services Research and Development Service, Veterans Health Administration, US Department of Veterans Affairs and Department of Psychiatry, University of Michigan Medical School, Ann Arbor, MD, USA.
\end{abstract}

Many of the most pressing health issues in the USA and worldwide require complex, multi-faceted solutions. Delivery of such solutions is often complicated by the need to reach and engage vulnerable populations facing multiple barriers to care. While the fields of quality improvement and implementation science have made valuable gains in the development and spread of individual strategies to improve evidence-based practice delivery, models for coordinated deployment of numerous strategies to simultaneously implement multiple evidence-based interventions in vulnerable populations are lacking. In this Perspective, we describe a model for this type of comprehensive research-practice translation effort: the Johns Hopkins ALACRITY Center for Health and Longevity in Mental Illness, which is focused on reducing premature mortality in the population with serious mental illness. We describe the Center's conceptual framework, which is built upon an integrated set of quality improvement and implementation science frameworks, provide an overview of the Center's organizational structure and core research-practice translation activities, and discuss our vision for how the Center may evolve over time. Lessons learned from this Center's efforts could inform models to address other critical health issues in vulnerable populations that require multi-component solutions at the policy, system, provider, and patient levels.

$\mathrm{J}$ Gen Intern Med 36(2):500-5

DOI: $10.1007 / \mathrm{s} 11606-020-06137-\mathrm{x}$

(c) Society of General Internal Medicine 2020

\section{INTRODUCTION}

Many of today's pressing health issues are what Charles West Churchman termed "wicked problems." Wicked problems

Received September 1, 2019

Accepted August 11, 2020

Published online August 31, 2020 have interacting causes, involve multiple decision-makers and clients with conflicting priorities and values, and require coordinated solutions across various systems and actors. ${ }^{1,2}$ While the interrelated fields of quality improvement and implementation science have made important gains in the development and spread of individual strategies to improve evidence-based practice delivery, for example, the quality improvement approaches LEAN and Six Sigma ${ }^{3}$ and facilitation implementation science strategies, ${ }^{4-7}$ wicked problems cannot be addressed by a single strategy. Rather, they require the coordinated use of multiple strategies to implement multiple evidence-based interventions. ${ }^{1,2}$ Concrete models for this type of multi-faceted effort are lacking. In this Perspective, we describe one such model: the ALACRITY Center for Health and Longevity in Mental Illness, a research-practice translation center designed to address the wicked problem of premature mortality among people with serious mental illness (SMI). People with SMI die 10-20 years earlier than the overall population, primarily due to cardiovascular disease..$^{8-13}$

This cardiovascular-related premature mortality is driven by multiple factors, including metabolic side effects of antipsychotic medications. ${ }^{14-17}$ Cognitive and communication impairments, lack of social support, and socioeconomic risks among people with SMI can impede the adoption of healthy behaviors and productive engagement with the healthcare system. ${ }^{18-27}$ Over $70 \%$ of primary care physicians (PCPs) believe that they should share responsibility with specialty mental health providers for treating physical health conditions and addressing tobacco smoking, diet, and exercise among people with SMI. ${ }^{28}$ But poor integration of general medical and specialty mental healthcare in the USA hinders such joint responsibility. Siloed medical training and delivery systems mean that PCPs may lack experience and comfort treating people with SMI, and physical healthcare is outside the 
purview of most mental health specialists. ${ }^{29,}{ }^{30}$ Health IT barriers and inadequate care coordination financing mechanisms limit the degree to which PCPs can collaborate with mental health providers to address cardiovascular risk in SMI.

Effective interventions to address cardiovascular disease and its risk factors and behaviors in people with SMI exist, but there are significant implementation gaps. ${ }^{31,32}$ While such gaps also exist in the general population, ${ }^{33-37}$ they are exacerbated among the population with SMI. Those with SMI - particularly the nearly $70 \%$ of people with SMI covered by Medicaid in the U.S. ${ }^{38}$ —often receive sub-optimal cardiovascular care. ${ }^{30}$ Tailored behavioral interventions have been shown in clinical trials to facilitate weight loss and tobacco smoking cessation among people with SMI, but are rarely implemented in real-world settings. ${ }^{32,}{ }^{39}$ These interventions are complex and can be challenging to implement with high fidelity and the required level of intensity. ${ }^{39}, 40$

The ALACRITY Center aims to develop scalable strategies to support widespread, high-fidelity implementation of effective interventions to reduce cardiovascular risk in SMI. In this Perspective, we provide a high-level overview of the Center. Protocols detailing the methodology of Center research projects will be published separately.

\section{ALACRITY CENTER FOR HEALTH AND LONGEVITY IN MENTAL ILLNESS}

\section{Mission}

In August 2018, the ALACRITY Center for Health and Longevity in Mental Illness at Johns Hopkins University was funded by the National Institute of Mental Health (NIMH) Advanced Laboratories for ACcelerating the Reach and Impact of Treatments for Youth and Adults with Mental Illness (ALACRITY) initiative. The Center's goals are to develop and test multi-component strategies to support the scale-up of evidence-based interventions to reduce cardiovascular risk in SMI. The strategies being tested by the Center integrate quality improvement and implementation science approaches. For this Center, we define quality improvement strategies as systematic processes leading to more effective organizational functioning, while implementation strategies are methods used to promote the uptake of specific evidence-based interventions. ${ }^{41,}{ }^{42}$ Both are crucial for sustaining new interventions in routine practice settings serving vulnerable populations like those with SMI. ${ }^{42}$

\section{Conceptual Framework}

The Center's work is guided by Proctor's conceptual model of implementation research. Figure 1 maps the Center's three core pilot projects onto the components of Proctor's model: evidencebased practices, implementation strategies, implementation outcomes, service/intervention outcomes, and health outcomes. The core projects (Fig. 1, box 1) focus on scaling up a tailored behavioral weight loss intervention ${ }^{43}$ (project 1); evidence- based tobacco smoking cessation treatment ${ }^{44-46}$ (project 2); and a care coordination intervention for hypertension, dyslipidemia, and diabetes mellitus (project 3$)^{47-51}$ These pilot studies will be conducted in a range of community mental health settings in Maryland (Fig. 1, box 1). The interventions will be implemented by mental health program staff, not research staff. Pilot project 1 uses a randomized controlled trial design and projects 2 and 3 use observational pre-post designs (as noted above, detailed study protocols will be published separately).

The goal of these projects is to identify promising multicomponent implementation strategies (Fig. 1, box 2) through pilot testing which will then be further refined and tested in large-scale hybrid implementation-effectiveness trials. ${ }^{52,}{ }^{53}$ All three projects include both quality improvement-oriented strategies designed to improve organizational functioning and implementation science-oriented strategies designed to support implementation of the specific evidence-based intervention of interest. For example, all three projects include quality improvementoriented organizational strategy meetings (OSMs). These meetings, led by a trained facilitator, bring together organizational leaders and front-line implementers to address barriers related to organizational functioning. ${ }^{54}$ All three projects include multimodality provider training, an implementation science-oriented strategy. (See Fig. 1, box 2, for the complete list of strategies tested in each project.)

For all three pilot projects, primary outcomes include the eight implementation research outcomes ${ }^{55}$ in Figure 1, box 3, as well as intervention outcomes, operationalized as measures of the degree to which each evidence-based practice was implemented, with fidelity, in real-world community mental health settings (Fig. 1, box 4). We will calculate costs for delivery of both the multi-component implementation strategy and the evidencebased intervention in each pilot study; this information will be critical to informing the development of financing mechanisms to support scale-up. Secondary outcomes include measures of consumers' cardiovascular health (Fig. 1, box 5).

\section{Center Organization}

Center researchers represent fields including primary care; specialty mental health; implementation science; quality improvement; human factors and systems engineering; organizational behavior; systems science; health services research, economics, and policy; epidemiology and biostatistics; behavioral science; and clinical trials. To facilitate effective collaboration, researchers are organized within cores, including administrative, training, community engagement, and methods cores (Fig. 2). Within the method core, researchers are organized into seven sub-cores with distinct responsibilities in support of the Center's research projects, e.g., implementation strategy development, data collection, and measurement.

\section{Cross-cutting Center Work}

The Center includes three cross-cutting components: innovative research methods to support scale-up of complex 


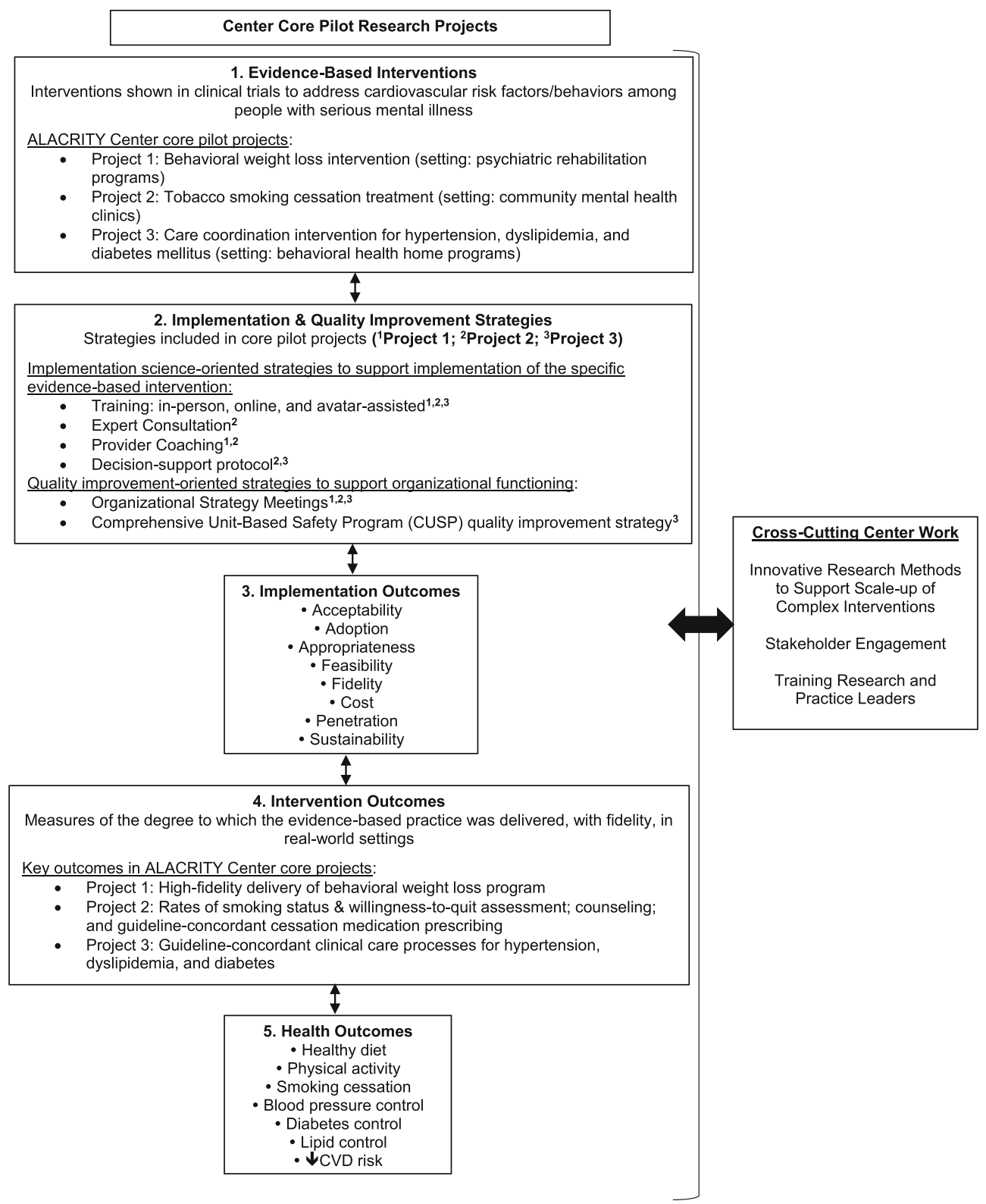

Figure 1 ALACRITY Center for Health and Longevity in Mental Illness Conceptual Framework.

interventions, stakeholder engagement, and training research and practice leaders (Fig. 1). Researchers spanning all the methods sub-cores come together to incubate innovative methods. For example, methods core researchers are collaborating on developing new methods for agent-based modeling of complex interventions. Pilot research project data will be incorporated into the models, and model results will then feedback into the research projects by forecasting how the implementation strategies being piloted might influence long-term intervention delivery and health outcomes. Such forecasts will help inform refinements to the strategies that will be tested in the post-pilot hybrid implementationeffectiveness trials.
Stakeholder engagement is also incorporated into all Center activities, led by the joint efforts of a stakeholder advisory board comprised of approximately 30 key practice and policy leaders (Fig. 2). The stakeholder advisory board includes leaders and front-line providers working in a range of community-based primary care and mental health settings; state and federal decision-makers, e.g., Medicaid program directors and other state/federal agency representatives; representatives of state and national mental health advocacy and professional organizations; peers; and people with SMI and family members (Fig. 2). The board strategically includes Maryland leaders as well as individuals representing national organizations who are well-positioned to support national scale-up in the future. 


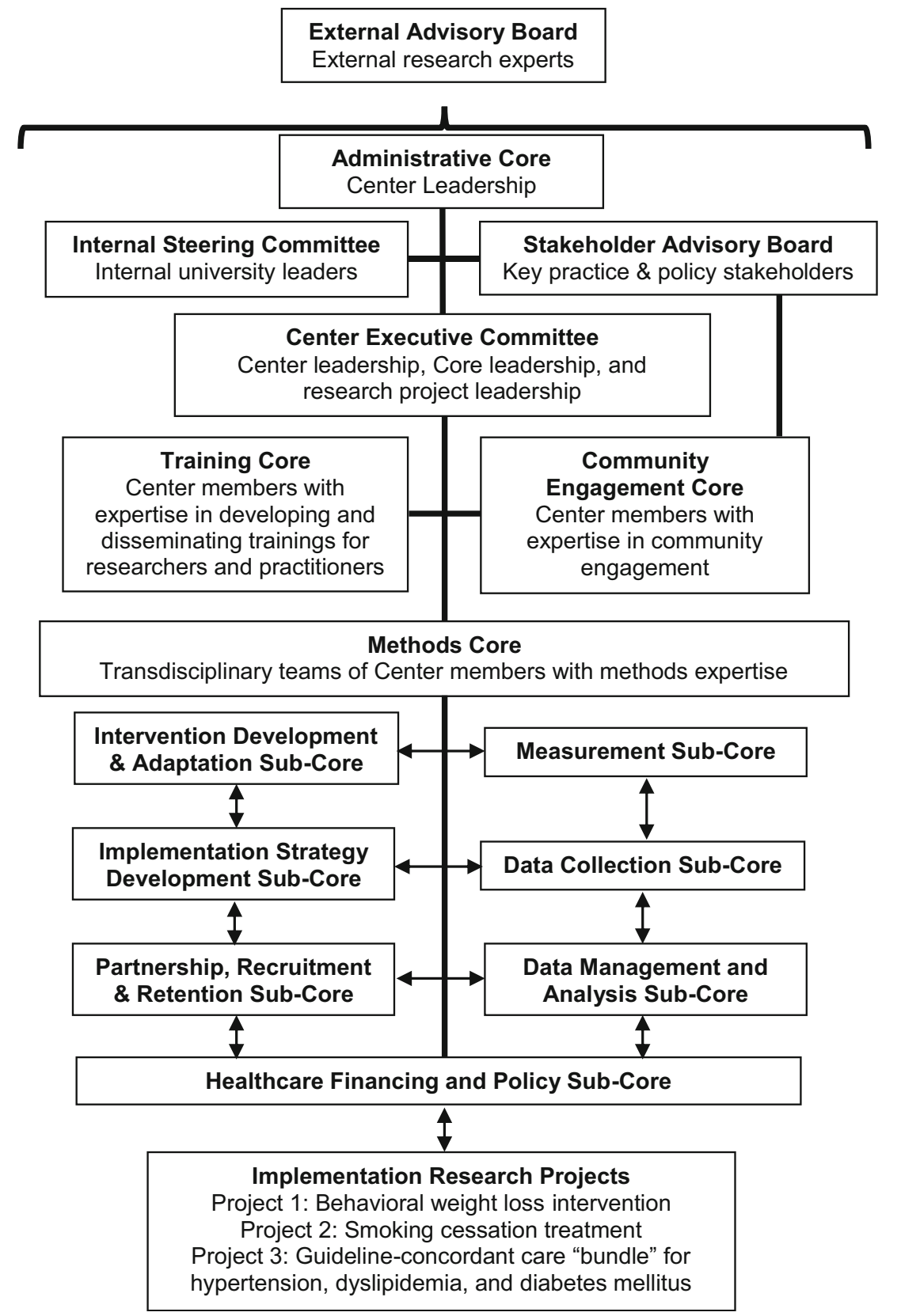

Figure 2 ALACRITY Center for Health and Longevity in Mental Illness organizational structure.

Stakeholder advisory board members are involved in all Center activities, including but not limited to identifying research priorities; providing input on recruitment, intervention, and data collection materials; developing and conducting trainings; reviewing pilot grant proposals submitted through the Center's seed funding program; mentoring seed funding awardees, each of whom is matched with a research mentor and a policy/practice mentor; and disseminating research findings.

The third key cross-cutting element of the Center's work is training the next generation of research and practice leaders focused on reducing premature mortality in SMI. This effort is led by the Center's training core (Fig. 2) and includes career development and mentorship programs for research trainees and junior faculty; seed funding awards; Center internships; and development and dissemination of trainings and other resources (e.g., fact sheets, policy memos) for a range of audiences.

\section{Moving Forward}

The immediate next step following completion of the Center's three pilot projects is to conduct large-scale, hybrid implementation-effectiveness trials rigorously evaluating the degree to which the strategies identified as promising in the pilots can facilitate high-fidelity intervention implementation resulting in improved cardiovascular health outcomes among people with SMI in real-world community mental health settings. Our vision is that Center activities around these 
interventions will then shift toward supporting national scaleout through activities such as train-the-trainer, certification, and accreditation programs and development and spread of financing mechanisms, e.g., insurer reimbursement.

In parallel with workaround bringing these initial three interventions to scale, we envision the Center moving forward with developing and testing strategies to support widespread implementation of additional evidence-based interventions, potentially including interventions that address conditions contributing to premature mortality in SMI other than cardiovascular disease (e.g., cancer, liver disease). Primary care has a central role to play in addressing the medical conditions that drive premature mortality in SMI, and we view strategies to bridge the specialty mental health and primary care sectors - such as the cardiovascular risk factor care coordination intervention in pilot project 3 - as one of the Center's core contributions to the field. In the long term, our vision is to support the development and adoption of models that allow PCPs to achieve their stated goal of sharing responsibility for the physical health of patients with SMI with specialty mental health providers. ${ }^{28}$ The Center's three initial pilot projects focus on implementation of cardiovascular risk reduction interventions in community mental settings. In the future, we anticipate developing and testing primary care setting-based strategies as well. Social services are included in pilot project 3's care coordination intervention, but otherwise, the Center's initial pilot projects do not address the social determinants of cardiovascular risk, a key limitation. In the future, we anticipate expanding our work to address social determinants, for example, through development of strategies for integrating financing and delivery of evidence-based healthcare and social services (e.g., supportive housing and employment) for people with SMI.

Moving forward, the ALACRITY Center will increasingly focus on policy issues. The Center's healthcare financing and policy core (Fig. 2) is currently laying the groundwork for this line of work by characterizing existing financing mechanisms and policy facilitators and barriers to nationwide scale-up of the three evidence-based interventions of interest in the Center's ongoing pilot research projects. Existing policies may impede scale-up. For example, state laws prohibiting Medicaid beneficiaries from receiving both a mental health and a physical health service in one day hinder delivery of coordinated cardiovascular care for people with SMI, and the prior authorization requirements for evidence-based smoking cessation medications imposed by some state Medicaid programs are a barrier to cessation treatment. ${ }^{56,57}$ In addition to removing policy barriers, new policies are likely needed: for example, insurance reimbursement mechanisms for tailored behavioral weight loss programs and two-way financing mechanisms that adequately reimburse both primary care and mental health providers for care coordination activities. The ALACRITY Center's future work will include a strong focus on development, evaluation, and dissemination - in close collaboration with our stakeholder advisory board—of such policies.
To the extent that our and other Centers funded through the ALACRITY initiative succeed in overcoming the researchpractice gap for "wicked problems" like premature mortality in SMI, this initiative could serve as a model for T2 translation for clinicians, researchers, funders, and community partners working to translate research into practice around a variety of complex medical and public health problems.

Corresponding Author: Emma E. McGinty, PhD, MS; Department of Health Policy and Management, Johns Hopkins Bloomberg School of Public Health, Baltimore, MD, USA (e-mail: bmcginty@jhu.edu).

Funding Information The authors received support from NIMH grant P5OMH115842

\section{Compliance with Ethical Standards:}

Conflict of Interest: The authors declare that they do not have a conflict of interest.

\section{REFERENCES}

1. Churchman CW. Wicked problems. Manag Sci. 1967;14(4):B141-B142.

2. Glasgow RE, Green LW, Taylor MV, Stange KC. An evidence integration triangle for aligning science with policy and practice. Am J Prev Med. 2012;42(6):646-654.

3. Glasgow JM, Scott-Caziewell JR, Kaboli PJ. Guiding Inpatient Quality Improvement: A Systematic Review of Lean and Six Sigma. Jt Comm J Qual Patient Saf. 2010;36(12):533-AP535.

4. Berta W, Cranley L, Dearing JW, Dogherty EJ, Squires JE, Estabrooks CA. Why (we think) facilitation works: insights from organizational learning theory. Implement Sci. 2015;10(1): 141.

5. Moussa L, Garcia-Cardenas V, Benrimoj SI. Change Facilitation Strategies Used in the Implementation of Innovations in Healthcare Practice: A Systematic Review. J Chang Manag. 2019;19(4):283-301.

6. Kilbourne AM, Goodrich DE, Lai Z, et al. Reengaging veterans with serious mental illness into care: preliminary results from a national randomized trial. Psychiatr Serv. 2014;66(1):90-93.

7. Kirchner JE, Ritchie MJ, Pitcock JA, Parker LE, Curran GM, Fortney JC. Outcomes of a partnered facilitation strategy to implement primary care-mental health. J Gen Intern Med. 2014;29(4):904-912.

8. Olfson M, Gerhard T, Huang C, Crystal S, Stroup TS. Premature mortality among adults with schizophrenia in the United States. JAMA Psychiatry. 2015;72(12):1172-1181.

9. Saha S, Chant D, McGrath J. A systematic review of mortality in schizophrenia: is the differential mortality gap worsening over time? Arch Gen Psychiatry. 2007;64(10):1123-1131.

10. Osborn DP, Levy G, Nazareth I, Petersen I, Islam A, King MB. Relative risk of cardiovascular and cancer mortality in people with severe mental illness from the United Kingdom's General Practice Rsearch Database. Arch Gen Psychiatry. 2007;64(2):242-249.

11. Osby U, Correia N, Brandt L, Ekbom A, Sparen P. Mortality and causes of death in schizophrenia in Stockholm county, Sweden, Schizophr Res. 2000;45(1-2):21-28.

12. Brown S. Excess mortality of schizophrenia. A meta-analysis. $\mathrm{Br} \mathrm{J}$ Psychiatry. 1997;171:502-508.

13. Daumit G, Anthony C, Ford DE, et al. Pattern of mortality in a sample of maryland residents with severe mental illness. Psychiatry Res. 2010;176(2-3):242-245.

14. McEvoy JP, Meyer JM, Goff DC, et al. Prevalence of the metabolic syndrome in patients with schizophrenia: Baseline results from the Clinical Antipsychotic Trials of Intervention Effectiveness (CATIE) Schizophrenia Trial and comparison with national estimates from NHANES III. Schizophr Res. 2005;80(1):19-32.

15. McIntyre RS, McCann SM, Kennedy SH. Antipsychotic metabolic effects: weight gain, diabetes mellitus, and lipid abnormalities. Can J Psychiatry/La Rev Can Psychiatr. 2001;46(3):273-281 
16. Meyer JM. Antipsychotics and metabolics in the post-CATIE era. Curr Top Behav Neurosci. 2010;4:23-42.

17. McGinty EE, Daumit GL. Epidemiology of Obesity. Psychiatr Ann. 2011;41(10):484-488.

18. Mueser KT, McGurk SR. Schizophrenia Lancet. 2004;363(9426):20632072.

19. Julian Chun-Chung C, Jaffee K, Snowden L. Racial/ethnic disparities in the use of mental health services in poverty areas. Am J Public Health. 2003;93(5):792-797.

20. Steadman HJ, Osher FC, Pamela Clark Robbins B, Case B, Samuels S. Prevalence of serious mental illness among jail inmates. Psychiatr Serv. 2009;60(6):761-765.

21. Lamb HR, Weinberger LE. Persons with severe mental illness in jails and prisons: a review. New Dir Ment Health Serv. 2001(90):29-49.

22. Calloway MO, Morrissey JP. Overcoming service barriers for homeless persons with serious psychiatric disorders. Psychiatr Serv (Washington, DC). 1998;49(12):1568-1572.

23. Draine J, Salzer MS, Culhane DP, Hadley TR. Role of social disadvantage in crime, joblessness, and homelessness among persons with serious mental illness. Psychiatr Serv. 2002;53(5):565-573.

24. Drake RE, Skinner JS, Bond GR, Goldman HH. Social Security And Mental Illness: Reducing Disability With Supported Employment. Health Aff. 2009;28(3):761-770.

25. Perkins R, Rinaldi M. Unemployment rates among patients with longterm mental health problems: A decade of rising unemployment. Psychiatr Bull. 2002;26(8):295-298.

26. Green MF. Cognitive impairment and functional outcome in schizophrenia and bipolar disorder. J Clin Psychiatry. 2006;67:3-8; discussion 3642 .

27. Buchanan J. Social support and schizophrenia: a review of the literature. Arch Psychiatr Nurs. 1995;9(2):68-76.

28. McGinty EE SE, Daumit GL. Serious Mental Illness in Primary Care: a National Physician Survey. Journal of General Internal Medicine. 2020:13.

29. Druss BG. Improving medical care for persons with serious mental illness: challenges and solutions. J Clin Psychiatry. 2007;68 Suppl 4:40-44.

30. McGinty EE, Baller J, Azrin ST, Juliano-Bult D, Daumit GL. Quality of medical care for persons with serious mental illness: A comprehensive review. Schizophr Res. 2015;165(2-3):227-235.

31. McGinty EE, Baller J, Azrin ST, Juliano-Bult D, Daumit GL. Interventions to address medical conditions and health-risk behaviors among persons with serious mental illness: a comprehensive review. Schizophr Bull. 2015;42(1):96-124.

32. Firth J, Siddiqi N, Koyanagi A, et al. The Lancet Psychiatry Commission: a blueprint for protecting physical health in people with mental illness. Lancet Psychiatry. 2019;6(8):675-712.

33. Abrams DB, Graham AL, Levy DT, Mabry PL, Orleans CT. Boosting Population Quits Through Evidence-Based Cessation Treatment and Policy. Am J Prev Med. 2010;38(3, Supplement):S351-S363.

34. Akers JD, Estabrooks PA, Davy BM. Translational Research: Bridging the Gap between Long-Term Weight Loss Maintenance Research and Practice. J Am Dietetic Assoc. 2010;110(10):1511-1522.e1513.

35. American Diabetes Association. 1. Improving Care and Promoting Health in Populations: Standards of Medical Care in Diabetes-2020. Diabetes Care. 2020;43(Supplement 1):S7-S13.

36. Arnold Sv, Goyal A, Inzucchi SE, et al. Quality of care of the initial patient cohort of the Diabetes Collaborative Registry®. J Am Heart Assoc. 2017;6(8):e005999.

37. National Committee for Quality Assurance. State of Health Care Quality. https://www.ncqa.org/report-cards/health-plans/state-of-health-carequality-report/, Accessed 20 February, 2020. 2018.

38. Khaykin E, Eaton Ww, Ford DE, Anthony CB, Daumit GL. Health insurance coverage among persons with schizophrenia in the United States. Psychiatr Serv (Washington, DC). 2010;61(8):830-834.

39. McGinty EE, Daumit GL. Interventions to Improve Somatic Health Outcomes in the the Population with Serious Mental Illness: A Comprehensive Review. Working paper 2014.
40. McGinty EE, Gudzune KA, Dalcin A, et al. Bringing an Effective Behavioral Weight Loss Intervention for People With Serious Mental Illness to Scale. Front Psychiatry. 2018;9.

41. Bauer MS, Damschroder L, Hagedorn H, Smith J, Kilbourne AM. An introduction to implementation science for the non-specialist. BMC Psychol. 2015;3(1):32.

42. Koczwara B, Stover AM, Davies L, et al. Harnessing the Synergy Between Improvement Science and Implementation Science in Cancer: A Call to Action. J Oncol Pract. 2018;14(6):335.

43. Daumit GL, Dickerson FB, Wang NY, et al. A Behavioral Weight Loss Intervention in Persons with Serious Mental Illness. NEJM. 2013;368(17):1594-1602.

44. Evins AE, Cather C, Laffer A. Treatment of tobacco use disorders in smokers with serious mental illness: toward clinical best practices. Harvard Rev Psychiatry. 2015;23(2):90.

45. Evins AE, Cather C, Pratt SA, et al. Maintenance treatment with varenicline for smoking cessation in patients with schizophrenia and bipolar disorder: a randomized clinical trial. JAMA. 2014;311(2):145-154.

46. Cather C, Pachas GN, Cieslak KM, Evins AE. Achieving smoking cessation in individuals with schizophrenia: special considerations. CNS Drugs. 2017;31(6):471-481.

47. American Diabetes Association. 3. Prevention or Delay of Type 2 Diabetes: Standards of Medical Care in Diabetes-2019. Diabetes Care. 2019;42(Suppl 1):S29.

48. American Diabetes Association. 5. Prevention or Delay of Type 2 Diabetes: Standards of Medical Care in Diabetes-2018. Diabetes Care. 2018;41(Suppl 1):S51.

49. Grundy SM, Stone NJ, Bailey AL, et al. 2018 AHA/ACC/AACVPR/ AAPA/ABC/ACPM/ADA/AGS/APhA/ASPC/NLA/PCNA guideline on the management of blood cholesterol: a report of the American College of Cardiology/American Heart Association Task Force on Clinical Practice Guidelines. J Am Coll Cardiol. 2019;73(24):e285-e350.

50. Arnett DK, Blumenthal RS, Albert MA, et al. 2019 ACC/AHA guidelineon the primary prevention of cardiovascular disease: a report of the American College of Cardiology/American Heart Association Task Force on Clinical Practice Guidelines. J Am Coll Cardiol. 2019;74(10):e177-e232.

51. American Diabetes Association. 9. Pharmacologic approaches to glycemic treatment: standards of medical care in diabetes-2019. Diabetes Care. 2019;42(Supplement 1):S90-S102

52. Curran GM, Bauer M, Mittman B, Pyne JM, Stetler C. Effectivenessimplementation hybrid designs: combining elements of clinical effectiveness and implementation research to enhance public health impact. Med Care. 2012;50(3):217-226.

53. Brown CH, Curran G, Palinkas LA, et al. An overview of research and evaluation designs for dissemination and implementation. Annu Rev Public Health. 2017;38:1-22.

54. Aarons GA, Ehrhart MG, Farahnak LR, Hurlburt MS. Leadership and organizational change for implementation (LOCI): a randomized mixed method pilot study of a leadership and organization development intervention for evidence-based practice implementation. Implement Sci. 2015;10(1): 11 .

55. Proctor E, Silmere H, Raghavan R, et al. Outcomes for Implementation Research: Conceptual distinctions, measurement challenges, and research questions. Manuscript under review.

56. DiGiulio A, Jump Z, Yu A, et al. State Medicaid coverage for tobacco cessation treatments and barriers to accessing treatments-United States, 2015-2017. Morb Mortal Wkly Rep. 2018;67(13):390.

57. Roby DH, Jones EE. Limits on same-day billing in Medicaid hinders integration of behavioral health into the medical home model. Psychol Serv. 2016;13(1): 110 .

Publisher's Note: Springer Nature remains neutral with regard to jurisdictional claims in published maps and institutional affiliations. 